\title{
ARTICLE OPEN \\ Efficacy of an occluding toothpaste on dentinal hypersensitivity over 14 days
}

\author{
Jonathan E. Creeth (D) ${ }^{1}$, Chhaju Goyal ${ }^{2}$, Jimmy Qaqish ${ }^{2}$, Robert Maclure ${ }^{3}$ and Jonathan S. Holt ${ }^{3}$
}

OBJECTIVES:: To evaluate a $0.454 \%$ stannous fluoride/5\% sodium tripolyphosphate (STP) toothpaste's ability to provide relief from dentinal hypersensitivity (DH) applied using 'focused brushing.'

MATERIALS AND METHODS:: In two randomised, examiner-blind, parallel-group studies, a $\mathrm{SnF}_{2} / \mathrm{STP}$ toothpaste was applied by brushing two selected sensitive teeth before 1 min whole-mouth brushing, compared to 1 min whole-mouth brushing only, with a negative control toothpaste. DH was assessed via evaporative (air) (Schiff scale) and tactile (Yeaple probe) stimuli after 7 and $14 \mathrm{~d}$ of twice-daily brushing.

RESULTS:: In total, 141 (Study 1)/142 (Study 2) participants were randomised. In Study 1, the test treatment significantly reduced $\mathrm{DH}$ at $7 / 14 \mathrm{~d}$ versus baseline (7/14 $\mathrm{d}$ Schiff difference: $-0.74[-0.84,-0.65] /-1.39[-1.54,-1.23]$; tactile: $6.00[4.88,7.13] / 15.30$

$[13.34,17.26])$; whereas the Control treatment did not (7/14 d Schiff difference $-0.03[-0.13,0.06] /-0.10[-0.25,0.06]$; tactile: 0.77 $[-0.36,1.90] / 0.77[-1.20,2.74])$. Differences between Test and Control were statistically significant $(p<0.0001$ all cases). In Study 2 , both treatments reduced $\mathrm{DH}$ compared to baseline by both measures, but there were no significant between-treatment differences. Toothpastes were generally well-tolerated.

DISCUSSION AND CONCLUSIONS:: Previous studies and Study 1 support $\mathrm{SnF}_{2} / 5 \%$ STP toothpaste efficacy; Study 2 results may have been influenced by placebo/Hawthorne effects. DH study design needs to, where possible, negate such effects.

BDJ Open (2021)7:26 ; https://doi.org/10.1038/s41405-021-00076-3

\section{INTRODUCTION}

Dentine can become exposed due to gum recession or enamel loss, leading to patent dentine tubules. ${ }^{1,2}$ According to the hydrodynamic theory, various stimuli--thermal, tactile, chemical, osmotic or evaporative, such as a hot drink or cold air--lead to movement of fluid in exposed dentinal tubules that stimulates nerve fibres in the pulp and causes a short, sharp pain. ${ }^{3-5}$ Up to $84 \%$ of the population report experiencing $\mathrm{DH}$ at some point in their life. ${ }^{6}$ While for some this pain is described as an occasional annoyance, for others it is a near-constant problem. ${ }^{7}$

Treatment of $\mathrm{DH}$ with daily-use oral care products generally focuses on one of two approaches. Nerve depolarisation, which blocks the pain response, can be achieved using potassium ions. ${ }^{8-10}$ These generally require at least $14 \mathrm{~d}$ before a benefit is established. ${ }^{11}$ Tubule occlusion--whereby exposed ends of dentine tubules are physically blocked to isolate the nerve from external stimuli--can be accomplished with the use of ingredients such as bioglasses, ${ }^{12,13}$ arginine plus particulate calcium ${ }^{14,15}$ and metal ions such as strontium $^{16,17}$ and stannous. ${ }^{17-19}$

Stannous fluoride $\left(\mathrm{SnF}_{2}\right)$ has long been used in anti-DH toothpaste formulations. Clinical studies demonstrate the efficacy of $\mathrm{SnF}_{2}$ toothpastes across a wide time-scale from after a single brushing to up to many months' use. ${ }^{18-24}$ Recently, studies have investigated how to reduce the time frame between using a $\mathrm{SnF}_{2}$ toothpaste and obtaining $\mathrm{DH}$ relief. Two routes have been explored: (i) the effect of application technique, specifically the effect of direct application prior to brushing, and (ii) the effect of formulation, specifically via optimisation of the base formulation polymer system.
Studies investigating direct application to sensitive areas prior to whole-mouth brushing have shown an advantage of brushing the sensitive teeth first for a defined period--christened 'focused brushing ${ }^{22-26}$--or by massaging the toothpaste into the affected teeth with a finger. ${ }^{16,27,28}$ Focused brushing studies have used a protocol in which the test product with an anti-DH ingredient has been applied directly to sensitive teeth prior to whole-mouth brushing, whereas the negative control product has been applied only by whole-mouth brushing. ${ }^{22,23}$ The protocol is therefore testing a regimen of test product/ application technique versus control product/application technique. This approach attempts to mimic a 'real-world' scenario where individuals suffering from $\mathrm{DH}$, who purchase a specific anti-DH toothpaste to treat it, would brush their sensitive teeth first, to ensure good contact of the anti-DH ingredients in the product with those sensitive areas. In contrast, those purchasing a conventional toothpaste would have no reason to apply it directly to their sensitive teeth (even if they suffered from DH), so would continue to brush normally.

The use of $\mathrm{SnF}_{2}$ as an anti-DH agent in a daily-use dentifrice brings formulation challenges. The stannous ion needs protecting from oxidation and/or hydrolysis during product storage prior to use. This can be achieved by formulating into an anhydrous, glycerine base. In addition, there are concerns about the staining potential of stannous ion-based toothpastes that have led to the inclusion of stain prevention and/or chemical cleaning agents, such as polyphosphates, into toothpaste formulations. ${ }^{25,26}$ The chelating agent sodium tripolyphosphate (STP) can be added to a

${ }^{1}$ GSK Consumer Healthcare, Weybridge, Surrey, UK; ${ }^{2}$ All Sum Research Centre Ltd, Mississauga, ON, Canada and ${ }^{3}$ Intertek Clinical Research Services, Ellesmere Port, Cheshire, UK Correspondence: Jonathan E. Creeth (jonathan.e.creeth@gsk.com)

Received: 22 October 2020 Revised: 12 January 2021 Accepted: 3 May 2021

Published online: 13 July 2021 
$\mathrm{SnF}_{2}$ toothpaste without compromising $\mathrm{DH}$ efficacy ${ }^{17}$ and has been shown to prevent stain build-up over 8 weeks. ${ }^{29}$

In vitro studies of such $\mathrm{SnF}_{2}$ formulations have provided insight into the mode of action. On exposure to the aqueous oral environment, tubule openings at the dentine surface are occluded by precipitates of stannous hydroxides and oxides together with particulates from the toothpaste and saliva-derived proteins and ions, leading to a reduction in tubular fluid flow. ${ }^{19,29-32}$ Studies involving optimising the formulation polymer system in this type of anhydrous base have shown that increasing the level of carbomer polymer can increase the rate of deposition/retention of stannous ion in vitro, while maintaining longer-term performance. $^{33}$

While two separate studies were undertaken, they both aimed to evaluate the ability of an experimental non-aqueous $0.454 \%$ $\mathrm{SnF}_{2} / 5 \%$ STP toothpaste with elevated carbomer level, applied by the focused brushing technique, to provide relief from $\mathrm{DH}$, as elicited by evaporative and tactile stimuli after 7 and 14-d of use, compared to a regular family fluoride toothpaste without known anti-DH or specific whitening agents.

\section{METHODS}

These two 14-day studies followed a randomised, examinerblind, two treatment-arm, parallel design and were stratified by maximum baseline Schiff sensitivity score and, for Study 2, by clinical site. Study 1 was conducted at a research facility in Canada; Study 2 was conducted at two sites (Ellesmere Port and Manchester, UK) of a UK-based research facility. Both were conducted in accordance with the Declaration of Helsinki and approved by independent research ethics committees before initiation (Study 1: Veritas IRB Inc. Reference 205201-2016-GSK-1; Study 2: North West--Liverpool Central Research Ethics Committee; Reference 16/NW/0065). These studies are registered at ClinicalTrials.org (Study 1: NCT02773758, Study 2: NCT02705716). Anonymised individual participant data and study documents can be requested for further research from www.clinicalstudydatarequest.com.

\section{Participants}

Both studies enrolled healthy participants aged $18-65$ y with no clinically significant or relevant abnormalities on oral examination. Participants were required to have a self-reported history of $\mathrm{DH}$ lasting more than 6 mo but not more than $10 y$ and $\geq 20$ natural teeth. At screening, eligible participants had at least two accessible teeth (incisors, canines or premolars) with signs of erosion, abrasion and/or facial/cervical gingival recession (EAR), a Modified Gingival Index ${ }^{34}$ score of 0 , a clinical mobility score $\leq 1$ and a positive response to a qualifying evaporative (air) assessment. At baseline (Day 0), eligible participants had a minimum of two accessible, non-adjacent teeth with signs of sensitivity, determined by a qualifying tactile stimulus threshold of $\leq 20 \mathrm{~g}$ and a Schiff sensitivity score $\geq 2$. $^{9}$

General exclusion criteria included pregnancy; breastfeeding; any known/suspected allergy or intolerance to study materials; participation in another clinical study or receipt of an investigational drug within $30 \mathrm{~d}$ or participation in a tooth-desensitising treatment study within 8 weeks prior to screening; a chronic debilitating disease that could affect study outcome; a xerostomiacausing condition/medication; daily use of medication that could affect pain perception; current use of antibiotics or use within 2 weeks prior to baseline visit.

General oral exclusions included tongue/lip piercings; dental implants; gross periodontal disease; exposed dentine with deep, defective or facial restorations; dental prophylaxis within 4 weeks, desensitising treatment or tooth bleaching within 8 weeks, scaling or root planing within $3 \mathrm{mo}$, periodontal disease treatment within 12 mo of screening. Specific dentition exclusions for test teeth included: current/recent caries, or reported treatment of decay, within 12 mo of screening; teeth used as abutments for fixed/ removable partial dentures; full crowns or veneers; orthodontic bands; cracked enamel or sensitive teeth with contributing aetiologies other than EAR; sensitive teeth not expected, in the investigator's opinion, to respond to treatment with an over-thecounter toothpaste.

\section{Procedures}

At the screening visit, each participant provided written informed consent to participate in the study before their demographic characteristics, medical history and use of concomitant medications were recorded. An oral soft tissue (OST) examination was then conducted. Each participant's dentition was assessed sequentially for evidence of EAR; gingival health status using the Modified Gingival Index; ${ }^{34}$ tooth mobility using a modification of the Miller scale; ${ }^{35}$ and sensitivity to an evaporative (air) stimulus (where a 'yes' response from the participant indicated sensitivity).

Eligible participants were supplied with a local market standard fluoride toothpaste (Study 1: Crest ${ }^{\oplus}$ Cavity Protection with 1000 ppm fluoride as sodium fluoride; Procter \& Gamble, Cincinnati, $\mathrm{OH}$, US; Canadian marketed product; Study 2: Signal ${ }^{\oplus}$ Family Protection with 1000 ppm fluoride as sodium monofluorophosphate [SMFP]; Unilever, London, UK; UK marketed product) and a toothbrush (Aquafresh ${ }^{\star}$ Clean Control [Everyday Clean], GSK Consumer Healthcare, Brentford, UK [GSKCH]) to use twice daily for 4-8 weeks between screening and baseline visits (acclimatisation period). While the lead-in toothpaste was different between the studies, differences between them with regard to relief of $\mathrm{DH}$ are not expected. ${ }^{36}$ First use of the toothpaste was carried out under supervision at the study site. Each use was recorded in a supplied diary.

At the baseline visit (Day 0), ongoing eligibility was assessed, any adverse events, incidents and changes to concomitant medications were recorded and compliance with the use of acclimatisation toothpaste was evaluated. Following an OST examination, hypersensitivity of the clinically eligible teeth identified at screening was evaluated by the participant's response to two separate, independent stimuli, in accordance with consensus guidelines in DH studies. ${ }^{37}$ The first DH assessment involved a tactile stimulus, administered by a constantpressure Yeaple probe. ${ }^{38}$ After at least $5 \mathrm{~min}$ (to ensure recovery from the tactile stimulus), teeth with a tactile threshold $\leq 20 \mathrm{~g}$ were evaluated for hypersensitivity to an evaporative (air) stimulus, assessed using the Schiff Sensitivity Scale. ${ }^{9}$ These two assessment techniques measure response to quite different types of dentine stimulus, to increase robustness of any observations of treatment effects. The Schiff examiner selected two non-adjacent hypersensitive teeth from those that met the qualifying assessments to be evaluated for the remainder of the study.

Before the study visits, participants refrained from all oral hygiene procedures and from taking analgesics for at least $8 \mathrm{~h}$, from eating and drinking for at least $4 \mathrm{~h}$ and from excessive alcohol consumption for $24 \mathrm{~h}$. Small sips of room temperature water were permitted for taking medication/relieving thirst if needed within $4 \mathrm{~h}$ before the visits but not within $1 \mathrm{~h}$. During the study, participants could not use any dental products other than those provided, nor any products for treating sensitive teeth. Use of dental floss was permitted for the removal of impacted food. Participants were to delay having any non-emergency dental treatment until after study completion.

Eligible participants were randomised to one of two treatment regimens according to a randomisation schedule provided by the study sponsor. Randomisation numbers were assigned in ascending numerical order as each participant was determined eligible. Randomisation was stratified by maximum baseline Schiff sensitivity score (either 2 or 3 ) of the two selected test teeth. The Test toothpaste contained $0.454 \% \mathrm{SnF}_{2}$ (1100 ppm fluoride) 
and 5\% STP. Participants applied a full ribbon of this toothpaste to a dry toothbrush then brushed each of the selected test teeth, followed by brushing the whole mouth for at least $60 \mathrm{~s}$. The Control toothpaste contained $0.76 \%$ SMFP (1000 ppm fluoride) (Colgate ${ }^{\circledast}$ Cavity Protection; Colgate-Palmolive, New York, NY, US; USA marketed product). Participants applied a full ribbon of this toothpaste to a dry toothbrush then brushed the whole mouth for at least $60 \mathrm{~s}$.

Both groups followed their brushing regimen twice a day (morning and evening) for $14 \mathrm{~d}$. First use of the study toothpaste was carried out under supervision at the study site. Compliance with use of the study toothpaste was assessed by review of the participant-completed diary cards. At each visit, participants underwent an OST examination before any clinical assessment of sensitivity. The dental examiners, study statistician, data management staff and other employees of the sponsor who could have influenced study outcomes were blinded to toothpaste allocation.

All study product tubes were overwrapped with white vinyl to blind participants to toothpaste assignment. The details of each brushing regimen were not discussed, i.e. that only the Test toothpaste included focused brushing, so participants were not made aware that the Control toothpaste regimen did not include focused brushing; hence they should not have known their group assignment.

\section{Assessments}

Clinical assessments of tooth sensitivity were made after 7 and 14 d of toothpaste use. As for the baseline assessments, two independent, stimulus-based clinical measures were used to assess $\mathrm{DH}$, in accordance with consensus guidelines, ${ }^{37}$

Firstly, a tactile stimulus was administered using a constantpressure (Yeaple) probe, ${ }^{38}$ which permitted application of a known force to the tooth surface. The greater the tactile threshold, the less sensitive the tooth. Testing began at a pressure of $10 \mathrm{~g}$ and was increased by $10 \mathrm{~g}$ with each successive challenge until either two consecutive 'yes' responses (with 'yes' indicating the stimulus caused pain or discomfort) were at the same pressure setting (recorded as the tactile threshold in grams) or the maximum force was reached. At baseline, maximum force was $20 \mathrm{~g}$; at subsequent visits, it was $80 \mathrm{~g}$.

Secondly, after a minimum 5-min recovery period, evaporative (air) sensitivity was assessed by directing a jet of air from a triple air dental syringe onto the exposed dentine surface from a distance of $\approx 1 \mathrm{~cm}$, with the test tooth surface isolated to prevent adjacent teeth or surrounding soft tissue being exposed to the stimulus. ${ }^{9}$ The examiner's assessment of the participant's response was recorded on the Schiff Sensitivity Scale (from $0=$ participant does not respond to air stimulus, to $3=$ participant responds to air stimulus, considers stimulus to be painful and requests discontinuation of the stimulus).

In Study 1, a single examiner performed all assessments. In Study 2, two examiners were employed across the two study centres, one who performed all the evaporative (air) assessments and one who performed all the tactile stimulus assessments.

\footnotetext{
Safety

Spontaneously reported adverse events (AEs) and any abnormalities in the OST examination were recorded from the start of use with the acclimatisation toothpaste at the screening visit until $5 \mathrm{~d}$ after the last use of study toothpaste. Treatmentemergent AEs (TEAEs) were reported for the safety population, which included all randomised participants who received the study toothpaste.
}

Data analysis

Sample size determination. Based on outcomes from previous sensitivity studies, ${ }^{21}$ for both Study 1 and 2 it was estimated that a sample of 60 participants per group would have an $\geq 90 \%$ power to detect a mean difference between the toothpastes of 0.35 units in Schiff sensitivity score (assuming a standard deviation [SD] of 0.5467 ) using a two-sided t-test of significance level 0.05 .

Efficacy analyses. Efficacy analyses were performed on a modified intent-to-treat $(\mathrm{mlTT})$ population, defined as all randomised participants who provided at least one post-baseline assessment of efficacy. The efficacy variables for analysis were the change from baseline at Days 7 and 14 (based on the change in the mean value of the two selected test teeth) in evaporative (air) sensitivity (using the Schiff Sensitivity Scale) and tactile sensitivity (using the tactile threshold). The primary endpoint was the change from baseline in Schiff sensitivity score at $14 \mathrm{~d}$. Change from baseline in Schiff sensitivity score was evaluated by analysis of covariance (ANCOVA), with treatment group and study site (Study 2 only) as factors, and baseline Schiff sensitivity score as a covariate. For tactile threshold, the mean baseline Schiff sensitivity score of the two selected test teeth was also included as a factor, with baseline tactile threshold score as a covariate. For all treatment groups, adjusted means and 95\% confidence intervals (Cls) were calculated.

The ANCOVA model assumptions for the analyses of Schiff sensitivity score were investigated and considered to be satisfied for both studies. For the tactile threshold data, the residual normality assumption was in doubt for Study 1 and failed for Study 2 , therefore, change in tactile threshold was also analysed by a non-parametric method (van Elteren test, adjusting for the maximum baseline Schiff Sensitivity scores). The results were compared with the ANCOVA results. For Study 1, the inferences from the two analyses were similar, therefore only the ANCOVA results are presented. For Study 2 only the van Elteren Test are presented for tactile threshold.

\section{RESULTS}

For Study 1,150 participants were screened and 141 were randomised to treatment (Fig. 1). The first participant was enrolled on 25 January 2016, the last completed the study on 11 March 2016. Table 1 shows baseline characteristics by group. The majority of participants in the safety population were female $(n=105$; $74.5 \%)$ and were white $(n=89 ; 63.1 \%)$; mean age was 47.9 y (SD: 10.39; range 18-64 y). Most participants were in Schiff strata ' 3 ': 108 (76.6\%).

For Study 2,409 participants were screened and 142 were randomised to treatment (Site 1 207/76, respectively; Site 2 202/66, respectively) (Fig. 1). The first participant was enrolled on 8 March 2016, the last participant completed the study on 20 May 2016. Table 1 shows baseline characteristics by group. The majority of participants in the safety population were female $(n=112 ; 78.9 \%)$ and were white $(n=119 ; 83.8 \%)$; mean age was 39.7 y (SD: 10.18 ; range 18-65y). Most participants were in Schiff strata ' 3 ': 131 (92.3\%).

The demographic characteristics of the treatment groups were similar across groups for the safety and ITT populations of both studies (Table 1).

\section{Efficacy}

In Study 1, there was a statistically significant change from baseline with the Test toothpaste at Days 7 and 14 (both $p<$ $0.0001)$, with decreases in Schiff sensitivity score of -0.74 (27.4\%) and $-1.39(51.1 \%)$ respectively (Table 2, Fig. 2$)$, and increases in tactile threshold score of $6.00 \mathrm{~g}(50.0 \%)$ and $15.30 \mathrm{~g}(128.6 \%)$ respectively (Table 2, Fig. 3). No significant differences were shown for the Control toothpaste on either measure. At both 7 and $14 \mathrm{~d}$, the change from baseline was statistically significantly greater for the Test toothpaste compared to the Control for both Schiff sensitivity and tactile threshold scores $(p<0.0001$ for all). 


\section{Study 1}

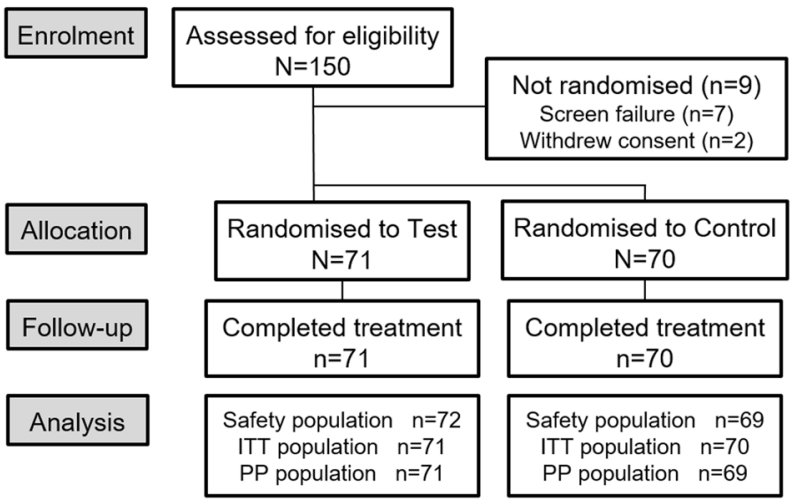

Study 2

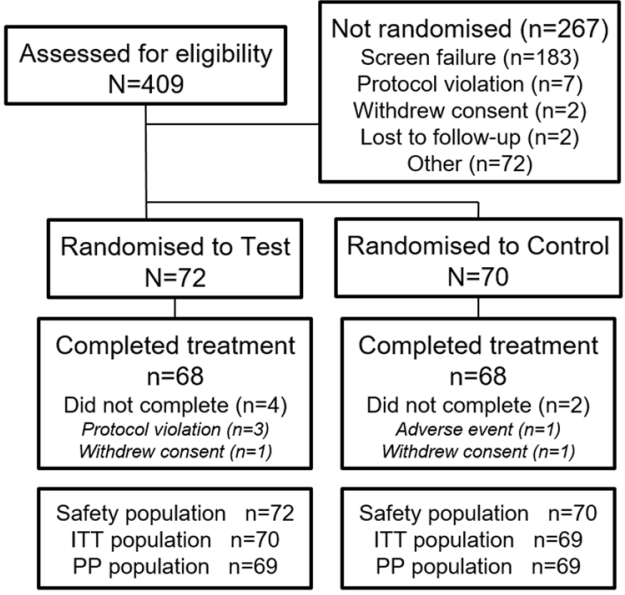

Fig. 1 Participant disposition throughout the study.

\begin{tabular}{|c|c|c|c|c|}
\hline \multirow[b]{2}{*}{ Characteristic } & \multicolumn{2}{|l|}{ Study 1} & \multicolumn{2}{|l|}{ Study 2} \\
\hline & Test $(n=72)$ & $\begin{array}{l}\text { Control ( } n \\
=69)\end{array}$ & Test $(n=72)$ & $\begin{array}{l}\text { Control }(n \\
=70)\end{array}$ \\
\hline \multicolumn{5}{|l|}{ Sex, $n(\%)$} \\
\hline Male & $17(23.6)$ & 19 (17.5) & $18(25.0)$ & $12(17.1)$ \\
\hline Female & $55(76.4)$ & $50(72.5)$ & $54(75.0)$ & $58(82.9)$ \\
\hline \multicolumn{5}{|l|}{ Age, y } \\
\hline Mean (SD) & $47.7(10.27)$ & $\begin{array}{l}48.0 \\
(10.58)\end{array}$ & 39.6 (10.89) & 39.9 (9.46) \\
\hline Range & $18-64$ & $19-64$ & $18-65$ & $18-65$ \\
\hline \multicolumn{5}{|l|}{ Race, $n$ (\%) } \\
\hline White & $40(55.6)$ & $49(71.0)$ & 59 (81.9) & 60 (85.7) \\
\hline $\begin{array}{l}\text { Black/African } \\
\text { American }\end{array}$ & $15(20.8)$ & $10(14.5)$ & 7 (9.7) & $7(10.0)$ \\
\hline Asian & $16(22.2)$ & $10(14.5)$ & $3(4.2)$ & $3(4.3)$ \\
\hline Multiple & $1(1.4)$ & 0 & $3(4.2)$ & 0 \\
\hline $\begin{array}{l}\text { Schiff strata 2, } \\
n(\%)\end{array}$ & 17 (23.6) & $16(23.2)$ & $6(8.3)$ & $5(7.1)$ \\
\hline $\begin{array}{l}\text { Schiff strata 3, } \\
n(\%)\end{array}$ & $55(76.4)$ & $53(76.8)$ & 66 (91.7) & $65(92.9)$ \\
\hline
\end{tabular}

In Study 2, there was also a statistically significant change from baseline with the Test toothpaste at Days 7 and 14 (both $p<0.0001$, with decreases in Schiff sensitivity score of -0.49 $(17.5 \%)$ and $-1.01(36.1 \%)$ respectively (Table 2, Fig. 2), and increases in tactile threshold score of $11.35 \mathrm{~g}$ (97.5\%) and $18.58 \mathrm{~g}$ (159.0\%) respectively (Table 2, Fig. 3). For the Control toothpaste, at both time points statistically significant differences were also shown in both Schiff sensitivity score (decreases of -0.54 [19.4\%] and -0.89 [31.4\%] for 7 and $14 \mathrm{~d}$ respectively) and tactile threshold score (increases of $10.15 \mathrm{~g}$ [85.5\%] and $18.56 \mathrm{~g}$ [156.7\%] for 7 and $14 \mathrm{~d}$ respectively) (Table $2, p<0.0001$ for all). There were no significant differences between the Test and Control toothpastes for either measure, at either time point.

Safety

In Study 1 there were three reported TEAEs, by three participants in the Test group, none of which was oral or considered treatment-related. All TEAEs were graded 'mild' and had resolved by the end of the study with no withdrawals due to a TEAE.

In Study 2, there were 19 TEAEs reported by 15 participants in the Test group, 30 TEAEs reported by 10 participants in the Control group. There were 10 oral TEAEs (eight participants) in the Test group, three oral TEAEs (three participants) in the Control group. TEAEs were mild or moderate in intensity and all but one (lost tooth restoration) had resolved by the end of the study. Only one TEAE was considered treatment-related: one incidence of mild oral mucosal exfoliation in the Test group. One TEAE led to participant withdrawal: root canal infection of moderate intensity in the Control group.

\section{DISCUSSION}

In the first of these two near-identically run studies (Study 1), a Test toothpaste containing $0.454 \% \mathrm{SnF}_{2}$ and $5 \%$ STP was shown to significantly improve $\mathrm{DH}$ compared to baseline, using two separate measures, when applied using a 'focused brushing' regimen. These differences were clinically meaningful on both measures by $14 \mathrm{~d}$ of use according to criteria of Orchardson et al., ${ }^{11}$ a reduction from baseline of at least $33 \%$. The Control toothpaste, containing no known anti-DH ingredients, performed as would be expected, in that there were no significant changes from baseline, meaning the Test treatment was significantly more effective in the two separate $\mathrm{DH}$ measures at both time points. These between-treatment differences were clinically meaningful from $7 \mathrm{~d}$, according to American Dental Association criteria of a difference between treatments of at least $20 \%$. $^{39}$

However, in Study 2, while a similar significant, clinically meaningful change from baseline was shown for the Test treatment on both measures, statistically significant changes were also shown for the Control treatment, with no significant betweentreatment differences.

The fact that such a different result was observed between studies with a near-identical design raises issues somewhat endemic to pain studies, even of treatments with accepted efficacy. That is, why do studies sometimes fail to show the expected difference between test and negative control treatments? There have been many studies of $\mathrm{SnF}_{2}$ toothpastes that confirm their efficacy in decreasing $\mathrm{DH}^{18-24}$ including those of 2 weeks' duration, $3 \mathrm{~d}$ and even immediate use. ${ }^{22-24}$ Therefore, the efficacy of the $\mathrm{SnF}_{2}$ toothpaste is hard to question. ${ }^{17}$ These current results mirror those of Parkinson et al. ${ }^{21}$ who used a similar study design and closely related test and negative control 


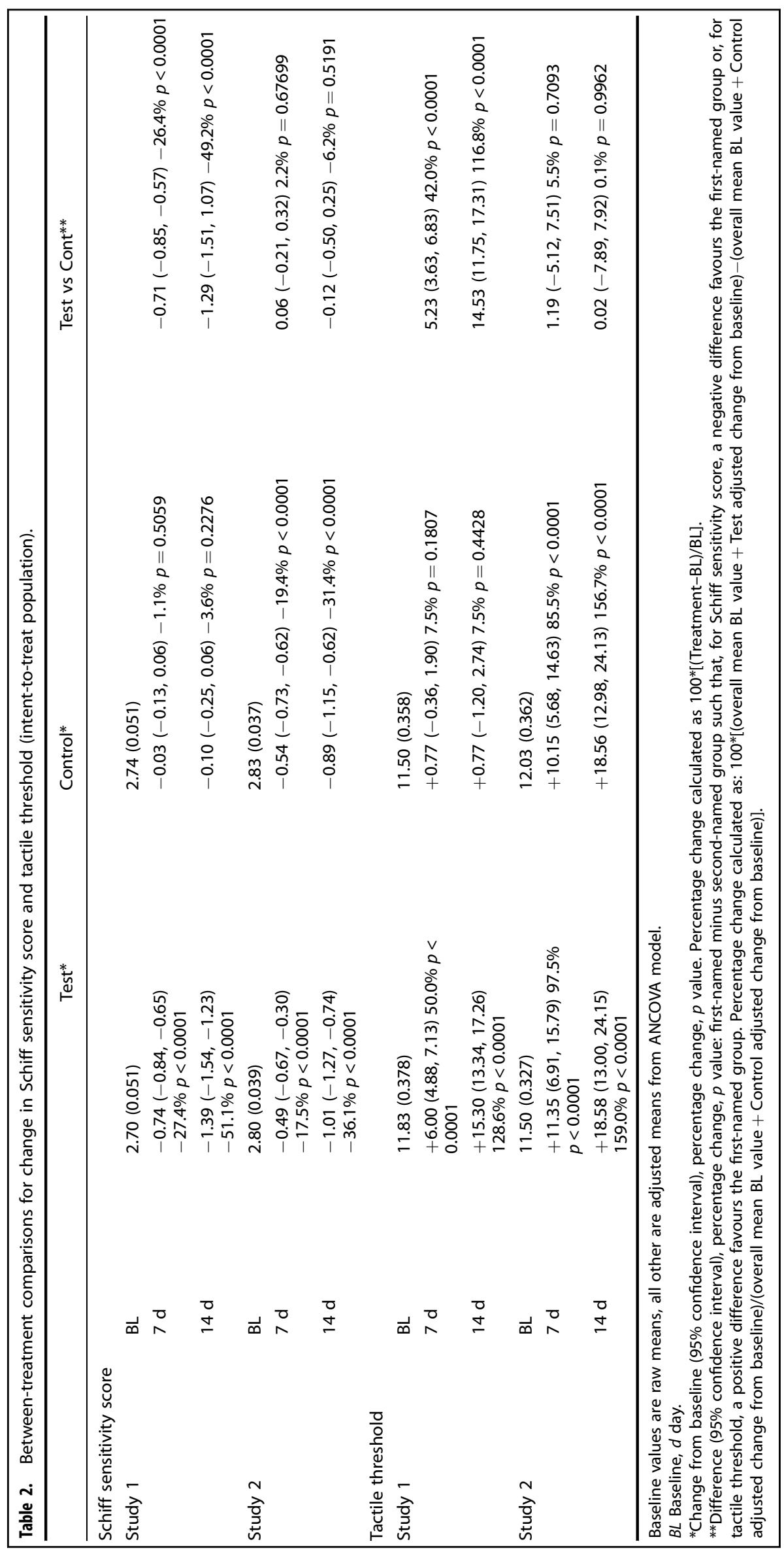




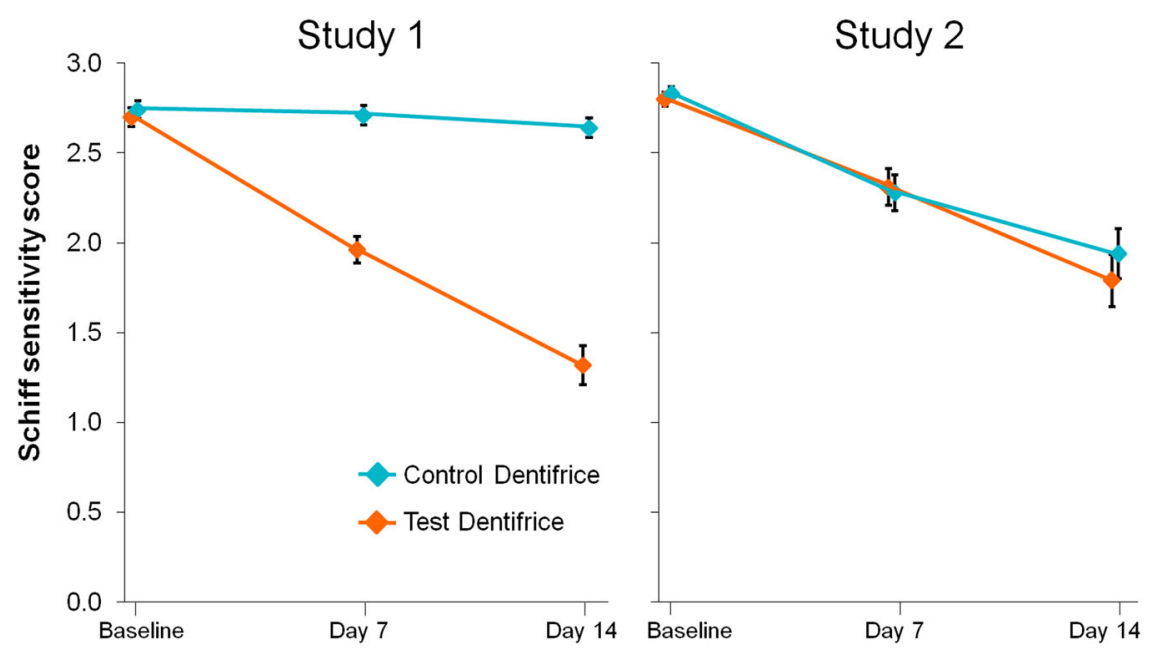

Fig. 2 Effect of treatments on Schiff sensitivity scores. Values are adjusted means ( \pm SE). Data are offset for clarity. Low values are favourable.

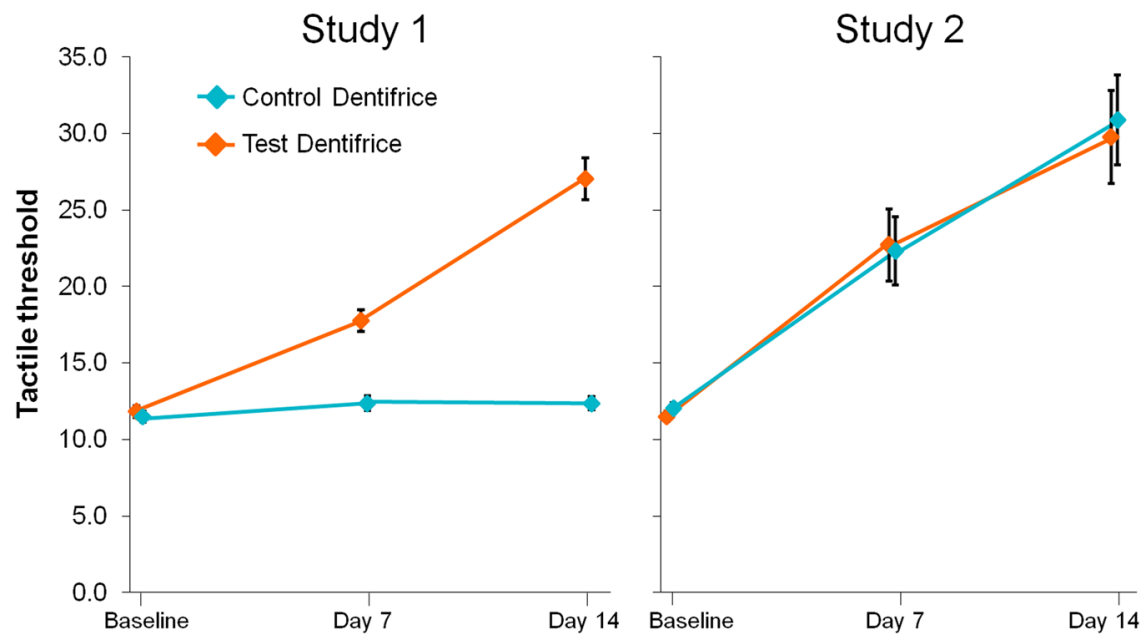

Fig. 3 Effect of treatments on Tactile threshold scores. Values are adjusted means ( \pm SE). Data are offset for clarity. Tactile threshold score range: $0-80 \mathrm{~g}$. High values are favourable.

toothpastes in three studies. While the test toothpaste was statistically significantly superior to the negative control by Day 14 in two of the studies, there were no such differences in the other trial. A substantial improvement in $\mathrm{DH}$ for the negative control appeared to be a key factor. In view of the existing body of evidence, therefore, the results of the current studies suggest that Study 2 was a 'false negative,' in which a true difference (as seen in Study 1) was, for some reason or reasons, not observed. Potential reasons for this outcome are discussed below.

Two factors are especially prevalent in pain studies such as this that use subjective assessments. One is the placebo effect--the response to a treatment of no known efficacy above that of not administering a treatment. ${ }^{40}$ The other is the Hawthorne effect--whereby behaviour changes occur due to the act of being observed in a study. ${ }^{41} \mathrm{DH}$ studies are known to be prone to both effects. ${ }^{42,43}$ West et al. ${ }^{43}$ suggested a range of $20-60 \%$ of treatment effects are due to a placebo response in conventional $\mathrm{DH}$ studies. In the three studies detailed in Parkinson et al., ${ }^{21}$ reductions from baseline for the negative control treatment ranged from $15-26 \%$ for Schiff sensitivity scores, with increases from baseline in tactile threshold scores ranging from $41-74 \%$. Here, respective percentage changes at 8 weeks for the Control toothpaste were $31.4 \%$ and $156.7 \%$, nearly identical to those with the Test toothpaste.
One of the few traits consistently found in those individuals liable to show a placebo response is a high baseline pain severity. ${ }^{44-46}$ This would suggest that the higher the degree of $\mathrm{DH}$ at screening, the greater the chance of improvement during the study, irrespective of treatment. This is of interest here because in Study 2 almost all the participants (overall 92.3\%) had the highest Schiff sensitivity score of ' 3 ,' compared to $76.6 \%$ of those in Study 1. While participants were stratified by baseline Schiff score to ensure an even number between treatments, this balances rather than reduces the problem, and it may simply be the case that differences between treatments are more apparent when the participant population has less severe initial $\mathrm{DH}$ than was prevalent in Study 2.

Another issue is the episodic nature of $\mathrm{DH}$, which means that it may resolve without treatment. ${ }^{43}$ Hence, though care was taken to only include those who had experienced $\mathrm{DH}$ for at least $6 \mathrm{mo}$ (though not necessarily in the test teeth), a natural resolution of $\mathrm{DH}$ could have been experienced by a sufficient proportion of participants in Study 2 to swamp product differences.

Treatment response may also be influenced by the Hawthorne effect. This arises because people tend to change their behaviour when they are being observed. An observational study of people with long term $\mathrm{DH}$ who used either an anti-DH or a non-anti-DH 
toothpaste, tracked them over 24 weeks and found improvements in both groups, suggesting that merely being in the study had brought about a change, despite participants knowing whether or not their toothpaste was targeting $\mathrm{DH}$ and not being assigned a toothpaste that was new to them. ${ }^{47}$

It is also known that those with $\mathrm{DH}$ may over-brush, thinking that the reason for experiencing $\mathrm{DH}$ is that they are not carrying out proper oral hygiene. ${ }^{43,48}$ Hence, changes in $\mathrm{DH}$ could be a response to being instructed on a gentler brushing regimen. ${ }^{43}$ In these current studies, while an explicit brushing style was not given, participants were told they could only brush their teeth for 1 min twice a day. If more of those in Study 2 than in Study 1 had been over-brushing, limiting their brushing may have reduced the amount of pain they experienced due to nerve stimulation by brushing action, regardless of toothpaste used. In addition, a reduction in brushing may have allowed greater undisturbed precipitation of calcium, phosphates and proteins from saliva onto the dentine surface, enhancing blockage of dentine tubules irrespective of dentifrice used.$^{43}$

Responses to treatment have also been shown to vary according to how a healthcare professional interacts with the participant. ${ }^{44}$ The release of endorphins elicited by positive emotional and behavioural responses can lead to a reduction in pain response, regardless of whether active treatment is given. ${ }^{49}$ Furthermore, if interactions with the assessor are on a very personable level, this can lead to the participant wanting to 'please' the assessor by, for instance, responding that treatment has had a greater effect on pain than in fact it has. ${ }^{43}$ This encourages using a fixed 'script' for DH studies. One difference between the studies was that in Study 1, there was a single examiner, but in Study 2, there were two, one for each measure. While both approaches are acceptable, to either limit interexaminer differences by only having one, or control for examiner error and bias by having two, it would be expected that if the reason for no treatment differences was due to examiner error in Study 2, this would only be reflected in only one of the measures, not both.

Benedetti et al. ${ }^{41}$ suggest that screening should also include an assessment of participant expectation and perceived treatment assignment to ascertain if this could contribute to the results. The differences in how participants were recruited between the studies could potentially have played a part in participant expectation. Differences in screening versus randomised participant numbers between study sites (Study 1: 150 screened, 141 randomised; Study 2: 409 screened, 142 randomised) were investigated and found to be due to differences in pre-screening criteria. In Study 1, participants were drawn from a heavily pre-screened database that had previously been registered, so there may have been a longer 'wait-list' period than in Study 2 where participants were recruited and screened in the same period. This shorter time between recruitment and study participation in Study 2 may have meant there were more participants than in Study 1 with an increased expectation of relief from $\mathrm{DH}$ due to study participation. This difference in recruitment approach is also believed to explain the high exclusion rate of participants at screening in Study 2, who were initially recruited based on a brief qualifying questionnaire with many failing at baseline due to the finding that they were using a banned medication or toothpaste (usually one that, while it may not have been explicitly labelled as such, did contain potential anti-DH ingredients) or that they did not have enough sensitivity at screening or baseline.

Having examined several of the factors that may have contributed to the differences shown between the two studies, it is of note that the design of these, and many other DH studies, included an acclimatisation period. This should have helped to overcome factors such as expectation of symptom relief and potential of spontaneous symptom relief, and acclimatised participants to being in a study and to possibly altering their brushing regimen. To account for any participants whose $\mathrm{DH}$ receded in this time, they were re-examined at baseline, with stricter criteria than screening, prior to dispensing of study treatment.

A final consideration as to why no differences were found in Study 2 is that the studies were only powered to provide a $90 \%$ chance of seeing a difference of the expected size as statistically significant, with the consequence of a one-in-ten chance that, even if the expected difference were observed, it would be found not statistically significant. This chance is in addition to natural biological variation in observed treatment difference, which will vary from the true difference from study to study.

Taken together, these experimental factors will combine to tend to reduce the degree of difference observed between an effective $\mathrm{DH}$ treatment and a negative control. It is, therefore, possible that the true benefit of the Test treatment is greater than that indicated by the study set.

Nevertheless, in terms of whether the evidence presented in this manuscript supports efficacy of the test product, we need also to consider the possibility that Study 1 showed an erroneously large difference between the products, i.e. it was a 'false positive,' and Study 2 was in fact the more accurate estimate of the true product difference. We believe this should be considered much less likely than the 'false negative' scenario described above, as the probability of the difference observed in study 1 not being real was below 1/10000, according to the statistical analysis. Randomisation and blinding were used to remove systematic bias that could have artificially exaggerated the true product difference.

In summary, studies such as those reported here are prone to a range of issues associated with subjective pain assessment. Although it cannot be stated with confidence from the data presented here in isolation that the Test $\mathrm{SnF}_{2}$ toothpaste treatment was superior to the Control toothpaste treatment in reducing $\mathrm{DH}$, the authors' conclusion is that the balance of evidence is in favour of efficacy of the $\mathrm{SnF}_{2}$ toothpaste treatment. This conclusion is based on the discussion above, showing that it is in practice much more likely that a 'false negative' has occurred in Study 2 than a 'false positive' in Study 1. This position is supported by data from a number of shorter- and longerterm studies clearly showing efficacy of $\mathrm{SnF}_{2}$ toothpastes against marketed control toothpastes, using similar clinical methodologies.

\section{ACKNOWLEDGEMENTS}

The authors would like to thank all study centre staff and study participants along with Farzana Sufi for her role in the development and interpretation of the study. Writing assistance was provided by Eleanor Roberts PhD, Beeline Science Communications, Ltd., funded by GSK Consumer Healthcare. Statistical analysis was provided by Darren Targett and Nan Wang, formerly of GSK Consumer Healthcare and by David Shaw, of GSK Consumer Healthcare.

\section{AUTHOR CONTRIBUTIONS}

C.G., J.Q., R.M., and J.H. led the design of the clinical studies; all authors contributed to elements of the study design. C.G., J.Q., R.M., and J.H. led the clinical research activities and data collection. J.C. led interpretation of results. All authors contributed to preparation and review of the manuscript, which was led by J.C.

\section{ADDITIONAL INFORMATION}

Competing interests: This study was funded by GSK Consumer Healthcare, of which J.C. is an employee. C.G. and J.Q. are employees of All Sum Research Centre Ltd, R.M. and J.H. were employees of Intertek Clinical Research Services at the time of the study, both of which companies have received funding from GSK Consumer Healthcare.

Publisher's note Springer Nature remains neutral with regard to jurisdictional claims in published maps and institutional affiliations. 


\section{REFERENCES}

1. Rimondini, L., Baroni, C. \& Carrassi, A. Ultrastructure of hypersensitive and nonsensitive dentine. A study on replica models. J. Clin. Periodontol. 12, 899-902 (1995).

2. West, N. X., Lussi, A., Seong, J. \& Hellwig, E. Dentin hypersensitivity: pain mechanisms and aetiology of exposed cervical dentin. Clin. Oral. Invest 17, S9-S19 (2013). Suppl 1.

3. Brännström M. A hydrodynamic mechanism in the transmission of pain producing stimuli through dentine. In D. J. Anderson (ed) Sensory Mechanisms in Dentine. Proceedings of a symposium held at the Royal Society of Medicine, London, September 24th, 1962. pp 73-79. Oxford: Pergamon Press, 1963.

4. Addy, M. Etiology and clinical implications of dentine hypersensitivity. Dent. Clin. North Am. 34, 502-514 (1990).

5. Canadian Advisory Board on Dentin Hypersensitivity. Consensus-based recommendations for the diagnosis and management of dentin hypersensitivity. J. Can. Dent. Assoc. (Tor.) 69, 221-226 (2003).

6. Cunha-Cruz J., Wataha J. C. The burden of dentine hypersensitivity. In Robinson P. G. (ed) Dentine Hypersensitivity. Developing a person-centred approach to oral health. Chp. 3. Oxford: Academic Press, 2014.

7. Gibson, B. et al. The everyday impact of dentine sensitivity: personal and functional aspects. Soc. Sci. Dent. 1, 11-20 (2010).

8. Markowitz, K. The original desensitizers: strontium and potassium salts. J. Clin. Dent. 20, 145-151 (2009).

9. Schiff, T. et al. Efficacy of a dentifrice containing potassium nitrate, soluble pyrophosphate, PVM/MA copolymer, and sodium fluoride on dentinal hypersensitivity: a twelve-week clinical study. J. Clin. Dent. 5, 87-92 (1994). Sp Iss.

10. Ling, T. Y. \& Gillam, D. G. The effectiveness of desensitizing agents for the treatment of cervical dentine sensitivity (CDS)-a review. J. West Soc. Periodontol. Periodontal Abstr. 44, 5-12 (1996).

11. Orchardson, R., Gangarosa, L. P. Sr, Holland, G. R. \& Pashley, D. H. Towards a standard code of practice for evaluating the effectiveness of treatments for hypersensitive dentine. Arch. Oral. Biol. 39, 121S-124S (1994).

12. Gendreau, L., Barlow, A. P. \& Mason, S. C. Overview of the clinical evidence for the use of NovaMin in providing relief from the pain of dentin hypersensitivity. J. Clin. Dent. 22, 90-95 (2011).

13. Pradeep, A. R. \& Sharma, A. Comparison of clinical efficacy of a dentifrice containing calcium sodium phosphosilicate to a dentifrice containing potassium nitrate and to a placebo on dentinal hypersensitivity: a randomized clinical trial. $J$. Periodontol. 81, 1167-1173 (2010).

14. Hughes, N. et al. A comparative clinical study investigating the efficacy of a test dentifrice containing $8 \%$ strontium acetate and $1040 \mathrm{ppm}$ sodium fluoride versus a marketed control dentifrice containing $8 \%$ arginine, calcium carbonate, and $1450 \mathrm{ppm}$ sodium monofluorophosphate in reducing dentinal hypersensitivity. J. Clin. Dent. 21, 49-55 (2010).

15. Bae, J. H., Kim, Y. K. \& Myung, S. K. Desensitizing toothpaste versus placebo for dentin hypersensitivity: a systematic review and meta-analysis. J. Clin. Periodontol. 42, 131-141 (2015).

16. Mason, S. et al. A comparative clinical study investigating the efficacy of a dentifrice containing $8 \%$ strontium acetate and $1040 \mathrm{ppm}$ fluoride in a silica base and a control dentifrice containing $1450 \mathrm{ppm}$ fluoride in a silica base to provide immediate relief of dentin hypersensitivity. J. Clin. Dent. 21, 42-48 (2010).

17. Makin, S. A. Stannous fluoride dentifrices. Am. J. Dent. 26, 3A-9A (2013).

18. Schiff, T., He, T., Sagel, L. \& Baker, R. Efficacy and safety of a novel stabilized stannous fluoride and sodium hexametaphosphate dentifrice for dentinal hypersensitivity. J. Contemp. Dent. Pr. 7, 1-10 (2006).

19. Parkinson, C. et al. The efficacy of an experimental dentifrice containing $0.454 \%$ $\mathrm{w} / \mathrm{w}$ stannous fluoride in providing relief from the pain of dentin hypersensitivity: An 8-week clinical study. Am. J. Dent. 26, 25A-31A (2013).

20. Schiff, T., Saletta, L., Baker, R., Winston, J. L. \& He, T. Desensitizing effect of a stabilized stannous fluoride/sodium hexametaphosphate dentifrice. Comp. Cont. Ed. Dent. 26, 35-40 (2005).

21. Parkinson, C. R. et al. Three randomized clinical trials to assess the short-term efficacy of anhydrous $0.454 \% \mathrm{w} / \mathrm{w}$ stannous fluoride dentifrices for the relief of dentin hypersensitivity. Am. J. Dent. 29, 25-32 (2016).

22. He, T. et al. Clinical evaluation of the fast onset and sustained sensitivity relief of a $0.454 \%$ stannous fluoride dentifrice compared to an $8.0 \%$ arginine-calcium carbonate-sodium monofluorophosphate dentifrice. Am. J. Dent. 24, 336-340 (2011).

23. He, T. et al. Fast onset sensitivity relief of a $0.454 \%$ stannous fluoride dentifrice. $J$. Clin. Dent. 22, 46-50 (2011).

24. He, T. et al. Rapid desensitizing efficacy of a stannous-containing sodium fluoride dentifrice. J. Clin. Dent. 22, 40-45 (2011). vol.

25. Sensabaugh, C. \& Sagel, M. E. Stannous fluoride dentifrice with sodium hexametaphosphate: Review of laboratory, clinical and practice-based data. J. Dent. Res 83, 70-78 (2009). Sp Iss 2.

26. $\mathrm{He}$, T. et al. A randomized clinical study to assess the extrinsic staining profiles of stannous- and triclosan containing dentifrices. Am. J. Dent. 23, 22B-26B (2010).
27. Nathoo, S. et al. Comparing the efficacy in providing instant relief of dentin hypersensitivity of a new dentifrice containing $8.0 \%$ arginine, calcium carbonate, and 1450 ppm fluoride to a benchmark desensitizing dentifrice containing $2 \%$ potassium ion and $1450 \mathrm{ppm}$ fluoride and to a control dentifrice with $1450 \mathrm{ppm}$ fluoride: a threeday clinical study in New Jersey, USA. J. Clin. Dent. 20, 123-130 (2009).

28. West, N., Newcombe, R. G. \& Hughes, N. A 3-day randomized clinical study investigating the efficacy of two toothpastes, designed to occlude dentine tubules, for the treatment of dentine hypersensitivity. J. Dent. 41, 187-194 (2013).

29. Nehme, M. et al. A randomized clinical study investigating the staining profile of an experimental stannous fluoride dentifrice. Am. J. Dent. 26, 32A-38A (2013). Sp Iss A.

30. Burnett, G. R., Willson, R. J. \& Lucas, R. A. In vitro studies investigating the dentin tubule-occlusion properties of an experimental anhydrous stannous fluoride dentifrice. Am. J. Dent. 26, 10A-14A (2013).

31. Burnett G. R. The effect of an experimental anhydrous stannous fluoride dentifrice on the acid resistance of dentin smear layers. Am. J. Dent. 26, 15A-18A (2013).

32. Earl, J. S. \& Langford, R. M. Physical and chemical characterization of the surface layers formed on dentin following treatment with an experimental anhydrous stannous fluoride dentifrice. Am. J. Dent. 26, 19A-24A (2013).

33. Khan S., Wilson S. Dentine permeability by an experimental formulation containing stannous fluoride. J. Dent. Res. 2017; 96(Spec Iss A): Abstract \#2122.

34. Lobene, R., Weatherford, T., Ross, N. M., Lamm, R. A. \& Menaker, L. A modified gingival index for use in clinical trials. Clin. Prev. Dent. 8, 3-6 (1986).

35. Laster, L., Laudenbach, K. W. \& Stoller, N. H. An evaluation of clinical tooth mobility measurements. J. Periodontol. 46, 603-607 (1975).

36. Gallob, J., Sufi, F., Amini, P., Siddiqi, M. \& Mason, S. A randomised exploratory clinical evaluation of dentifrices used as controls in dentinal hypersensitivity studies. J. Dent. 64, 80-87 (2017).

37. Holland, G. R. et al. Guidelines for the design and conduct of clinical trials on dentine hypersensitivity. J. Clin. Periodontol. 24, 808-813 (1997).

38. Polson, A. M., Caton, J. G., Yeaple, R. N. \& Zander, H. A. Histological determination of probe tip penetration into gingival sulcus of humans using an electronic pressure-sensitive probe. J. Clin. Periodontol. 7, 479-488 (1980).

39. American Dental Association Acceptance Program Guidelines: Products for the treatment of dentinal hypersensitivity. American Dental Association, Council on Scientific Affairs, 2012.

40. Kirsch, I. The placebo effect revisited: lessons learned to date. Complement Ther. Med 21, 102-104 (2013).

41. Benedetti, F., Carlino, E. \& Piedimonte, A. Increasing uncertainty in CNS clinical trials: the role of placebo, nocebo, and Hawthorne effects. Lancet Neurol. 15, 736-747 (2016).

42. Addy M., Embery G., Edgar W. M., Orchardson R. (eds), Tooth wear and sensitivity. Clinical advances in restorative dentistry. Martin Dunitz, London, 2000.

43. West, N. X., Addy, M., Jackson, R. J. \& Ridge, D. B. Dentin hypersensitivity and the placebo response. A comparison of the effect of strontium acetate, potassium nitrate and fluoride dentifrices. J. Clin. Periodontol. 24, 209-215 (1997).

44. Häuser, W. et al. Systematic review: placebo response in drug trials of fibromyalgia syndrome and painful peripheral diabetic neuropathy-magnitude and patient-related predictors. Pain 152, 1709-1717 (2011).

45. Irizarry, M. C. et al. Predictors of placebo response in pooled lamotrigine neuropathic pain clinical trials. Clin. J. Pain. 25, 469-476 (2009).

46. Zhang, W., Robertson, J., Jones, A. C., Dieppe, P. A. \& Doherty, M. The placebo effect and its determinants in osteoarthritis: meta-analysis of randomised controlled trials. Ann. Rheum. Dis. 67, 1716-1723 (2008).

47. Leight R., Bowman J., Barlow A. Dentinal hypersensitivity: evidence for a "no treatment" effect. J. Dent. Res. 87, Abstract No 3498 (2008).

48. Bradnock, G. et al. Dental attitudes and behaviours in 1998 and implications for the future. Br. Dent. J. 190, 228-284 (2001).

49. Trowbridge, H. O. \& Silver, D. R. A review of current approaches to in-office management of tooth hypersensitivity. Dent. Clin. North Am. 34, 561-582 (1990). Attribution 4.0 International License, which permits use, sharing, adaptation, distribution and reproduction in any medium or format, as long as you give appropriate credit to the original author(s) and the source, provide a link to the Creative Commons license, and indicate if changes were made. The images or other third party material in this article are included in the article's Creative Commons license, unless indicated otherwise in a credit line to the material. If material is not included in the article's Creative Commons license and your intended use is not permitted by statutory regulation or exceeds the permitted use, you will need to obtain permission directly from the copyright holder. To view a copy of this license, visit http://creativecommons. org/licenses/by/4.0/.

(c) The Author(s) 2021 